\title{
TOPOLOGY OF FACTORED ARRANGEMENTS OF LINES
}

\author{
LUIS PARIS
}

(Communicated by Peter Li)

\begin{abstract}
A real arrangement of affine lines is a finite family $\mathscr{A}$ of lines in $\mathbf{R}^{2}$. A real arrangement $\mathscr{A}$ of lines is said to be factored if there exists a partition $\Pi=\left(\Pi_{1}, \Pi_{2}\right)$ of $\mathscr{A}$ into two disjoint subsets such that the OrlikSolomon algebra of $\mathscr{A}$ factors according to this partition. We prove that the complement of the complexification of a factored real arrangement of lines is a $K(\pi, 1)$ space.
\end{abstract}

\section{INTRODUCTION}

Let $\mathbf{K}$ be a field, and let $V$ be a vector space over $\mathbf{K}$. An arrangement of (affine) hyperplanes in $V$ is a finite family $\mathscr{A}$ of (affine) hyperplanes of $V$. An arrangement of (affine) lines is an arrangement of hyperplanes in a 2dimensional vector space $V=\mathbf{K}^{2}$. An arrangement $\mathscr{A}$ of hyperplanes is said to be real (resp. complex) if $\mathbf{K}=\mathbf{R}$ is the field of real numbers (resp. if $\mathbf{K}=\mathbf{C}$ is the field of complex numbers). The complexification of a hyperplane $H$ of $\mathbf{R}^{l}$ is the hyperplane $H_{\mathrm{C}}$ of $\mathrm{C}^{l}$ having the same equation as $H$. The complexification of a real arrangement $\mathscr{A}$ of hyperplanes in $\mathbf{R}^{l}$ is the arrangement $\mathscr{A}_{\mathbf{C}}=\left\{H_{\mathbf{C}} \mid\right.$ $H \in \mathscr{A}\}$ in $\mathbf{C}^{l}$.

Let $\mathscr{A}$ be a complex arrangement of hyperplanes in $V=\mathrm{C}^{l}$. The complement of $\mathscr{A}$ is the connected submanifold

$$
M(\mathscr{A})=V-\left(\bigcup_{H \in \mathscr{A}} H\right)
$$

of $V$. We say that $\mathscr{A}$ is a $K(\pi, 1)$ arrangement if $M(\mathscr{A})$ is a $K(\pi, 1)$ space. We say that a real arrangement $\mathscr{A}$ of hyperplanes is a $K(\pi, 1)$ arrangement if its complexification $\mathscr{A}_{C}$ is a $K(\pi, 1)$ arrangement. Yet, only two classes of real $K(\pi, 1)$ arrangements of hyperplanes are known. These are the simplicial arrangements (see [De]) and supersolvable arrangements (see [Te1]). Other examples of real $K(\pi, 1)$ arrangements appear in [Fa] and in [JS].

Our aim in this paper is to produce a new class of real $K(\pi, 1)$ arrangements: "factored arrangements of lines". This class contains supersolvable arrangements of lines (see [Ja]).

We refer to [FR] for a good exposition on $K(\pi, 1)$ arrangements.

Received by the editors April 12, 1993.

1991 Mathematics Subject Classification. Primary 52B30. 
Let $\mathscr{A}$ be an arrangement of hyperplanes. The intersection poset of $\mathscr{A}$ is the ranked poset $\mathscr{L}(\mathscr{A})$ consisting of all nonempty intersections of elements of $\mathscr{A}$ ordered by reverse inclusion. $V=\bigcap_{H \in \varnothing} H$ is assumed to be the smallest element of $\mathscr{L}(\mathscr{A})$. For $X \in \mathscr{L}(\mathscr{A})$, we set

$$
\mathscr{A}_{X}=\{H \in \mathscr{A} \mid H \supseteq X\} .
$$

A partition $\Pi=\left(\Pi_{1}, \ldots, \Pi_{l}\right)$ of $\mathscr{A}$ into $l$ disjoint nonempty subsets is called independent if, for any choice of hyperplanes $H_{i} \in \Pi_{i}(i=1, \ldots, l)$, the subspace $H_{1} \cap \cdots \cap H_{l}$ is nonempty and its rank is $l$ in $\mathscr{L}(\mathscr{A})$. If $X \in \mathscr{L}(\mathscr{A})$, then $\Pi$ induces a partition $\Pi_{X}$ of $\mathscr{A}_{X}$ whose blocks are the nonempty subsets $\Pi_{i} \cap \mathscr{A}_{X}$. A partition $\Pi=\left(\Pi_{1}, \ldots, \Pi_{l}\right)$ of $\mathscr{A}$ is a factorization (or a nice partition) if

(1) $\Pi$ is independent;

(2) if $X \in \mathscr{L}(\mathscr{A})-\{V\}$, then $\Pi_{X}$ has at least a block which is a singleton. If $\mathscr{A}$ is an arrangement of lines, then any factorization of $\mathscr{A}$ has to be a partition $\Pi=\left(\Pi_{1}, \Pi_{2}\right)$ of $\mathscr{A}$ into two disjoint subsets (see [Te2]). We say that an arrangement $\mathscr{A}$ of hyperplanes is factored if $\mathscr{A}$ has a factorization.

Factored arrangements have been introduced and investigated by Falk, Jamba, and Terao [FJ, Te2]. One of the main results concerning these arrangements is the following theorem due to Terao [Te2].

The homogeneous component $A^{1}(\mathscr{A})$ of the Orlik-Solomon algebra $A(\mathscr{A})$ of an arrangement $\mathscr{A}$ of hyperplanes can be viewed as a free Z-module spanned by the hyperplanes of $\mathscr{A}$ (see [OS]). For $\mathscr{B} \subseteq \mathscr{A}$, we denote by $B(\mathscr{B})$ the submodule of $A^{1}(\mathscr{A})$ spanned by the elements of $\mathscr{B}$.

Theorem 1 (Terao [Te2]). Let $\mathscr{A}$ be an arrangement of hyperplanes. Let $\Pi=$ $\left(\Pi_{1}, \ldots, \Pi_{l}\right)$ be a partition of $\mathscr{A}$. The Orlik-Solomon algebra of $\mathscr{A}$, viewed as a graded Z-module, factors as

$$
A(\mathscr{A})=\left(\mathbf{Z} \oplus B\left(\Pi_{1}\right)\right) \otimes \cdots \otimes\left(\mathbf{Z} \oplus B\left(\Pi_{l}\right)\right)
$$

if and only if $\Pi$ is a factorization.

Our goal in this paper is to prove the following theorem.

Theorem 2. If $\mathscr{A}$ is a factored real arrangement of lines, then $\mathscr{A}$ is a $K(\pi, 1)$ arrangement.

Example. Consider the arrangement $\mathscr{A}$ shown in Figure 1. Set $\Pi_{1}=$ $\left\{l_{1}, l_{2}, l_{3}, l_{4}\right\}$ and $\Pi_{2}=\left\{l_{5}, l_{6}, l_{7}, l_{8}\right\}$. Then $\Pi=\left(\Pi_{1}, \Pi_{2}\right)$ is a factorization of $\mathscr{A}$. Note that this arrangement is neither simplicial nor supersolvable.

A direct consequence of Theorem 2 is the following corollary. Recall that an arrangement $\mathscr{A}$ of hyperplanes is said to be central if $\bigcap_{H \in \mathscr{A}} H \neq \varnothing$.

Corollary. Let $\mathscr{A}$ be a real and central arrangement of hyperplanes. Assume that the rank of $\mathscr{L}(\mathscr{A})$ is 3. If $\mathscr{A}$ is a factored arrangement, then $\mathscr{A}$ is a $K(\pi, 1)$ arrangement.

Proof. Let $\Pi=\left(\Pi_{1}, \Pi_{2}, \Pi_{3}\right)$ be a factorization of $\mathscr{A}$. One may assume that $\mathscr{A}$ is an arrangement in $\mathbf{R}^{3}$, that $\bigcap_{H \in \mathscr{A}} H=\{0\}$, and that $\Pi_{3}$ is a singleton $\left\{H_{0}\right\}$. Let $K_{0}$ be an (affine) hyperplane of $\mathbf{R}^{3}$ parallel to $H_{0}$ and different 


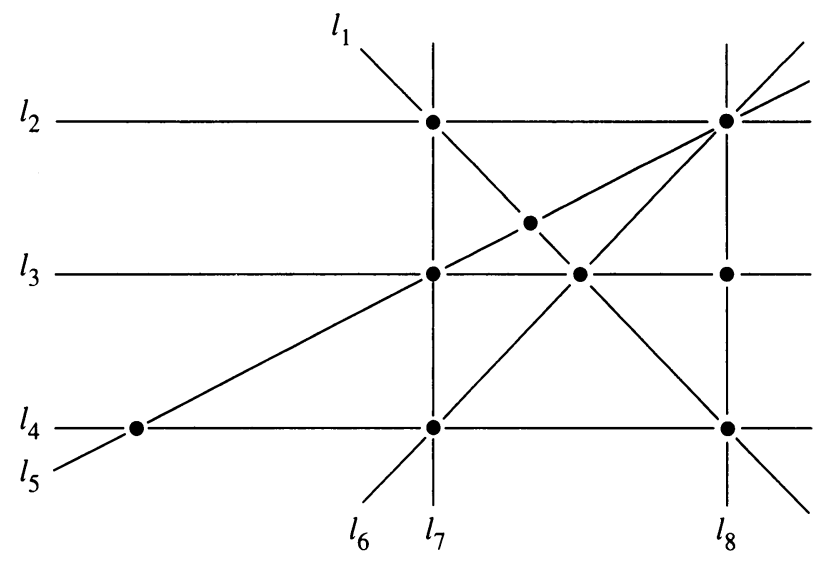

FIGURE 1

from $H_{0}$. Set

$$
\begin{aligned}
& \widetilde{\mathscr{A}}=\left\{H \cap K_{0} \mid H \in \mathscr{A}-\left\{H_{0}\right\}\right\}, \\
& \widetilde{\Pi}_{1}=\left\{H \cap K_{0} \mid H \in \Pi_{1}\right\}, \\
& \widetilde{\Pi}_{2}=\left\{H \cap K_{0} \mid H \in \Pi_{2}\right\} .
\end{aligned}
$$

Then $\widetilde{\mathscr{A}}$ is a real arrangement of lines in $K_{0}$, the partition $\widetilde{\Pi}=\left(\widetilde{\Pi}_{1}, \widetilde{\Pi}_{2}\right)$ is a factorization of $\widetilde{\mathscr{A}}$, and $M\left(\mathscr{A}_{\mathrm{C}}\right)$ is homeomorphic to $\mathrm{C}^{*} \times M\left(\widetilde{\mathscr{A}}_{\mathrm{C}}\right)$ (see [OT, Proposition 5.1.1]). So, $M\left(\mathscr{A}_{\mathrm{C}}\right)$ is a $K(\pi, 1)$ space since, by Theorem 2, $M\left(\widetilde{\mathscr{A}_{\mathrm{C}}}\right)$ is a $K(\pi, 1)$ space.

The proof of Theorem 2 is a direct application of Falk's weight test for a real arrangement of lines to be $K(\pi, 1)$ (see [Fa]).

Section 2 is divided into two subsections. In $\S 2.1$ we state Falk's weight test (Theorem 3). In $\S 2.2$ we prove Theorem 2.

\section{Proof of Theorem 2}

Throughout this section $\mathscr{A}$ is assumed to be an arrangement of affine lines in $V=\mathbf{R}^{2}$.

2.1. Falk's weight test for $K(\pi, 1)$ arrangements. The lines of $\mathscr{A}$ subdivide $V$ into facets. The support $|f|$ of a facet $f$ is the smallest affine subspace of $V$ containing $f$. Every facet is open in its support. We denote by $\bar{f}$ the closure of $f$ in $V$. There is a partial order on the set of facets defined by $f \leq g$ if $f \subseteq \bar{g}$. 0-dimensional facets are called vertices, 1-dimensional facets are called edges, and 2-dimensional facets are called faces.

Let $\Gamma(\mathscr{A})$ denote the planar 2-complex consisting of the bounded facets. We denote by $\Gamma^{(i)}(A)$ its $i$-skeleton $(i=0,1,2)$. A corner of $\Gamma(\mathscr{A})$ is a chain $(v<f)$ with $v \in \Gamma^{(0)}(\mathscr{A})$ and $f \in \Gamma^{(2)}(\mathscr{A})$. We denote by Corn $(\mathscr{A})$ the set of corners. A system of weights on $\Gamma(\mathscr{A})$ is a function $\Omega$ : $\operatorname{Corn}(\mathscr{A}) \rightarrow \mathbf{R}^{+}=$ $[0,+\infty[$.

Let $v \in \Gamma^{(0)}(\mathscr{A})$. The link graph of $\Gamma(\mathscr{A})$ at $v$ is the graph $\Lambda_{v}$ defined as follows.

(1) The vertices of $\Lambda_{v}$ are the chains $(v<e)$ with $e \in \Gamma^{(1)}(\mathscr{A})$. 
(2) The edges of $\Lambda_{v}$ are the chains (or corners) $(v<f)$ with $f \in \Gamma^{(2)}(\mathscr{A})$.

(3) An edge $(v<f)$ is incident with a vertex $(v<e)$ if $v<e<f$. Let $\gamma=\left(\left(v<e_{0}\right),\left(v<e_{1}\right), \ldots,\left(v<e_{n}\right)\right)$ be a path in $\Lambda_{v}$. For $j=1, \ldots, n$, let $\left(v<f_{j}\right)$ be the edge of $\Lambda_{v}$ incident with $\left(v<e_{j-1}\right)$ and $\left(v<e_{j}\right)$. For a given system of weights $\Omega$ : $\operatorname{Corn}(\mathscr{A}) \rightarrow \mathbf{R}^{+}$, we define the weight of $\gamma$ to be

$$
\Omega(\gamma)=\sum_{j=1}^{n} \Omega\left(v<f_{j}\right)
$$

A path $\gamma=\left(\left(v<e_{0}\right),\left(v<e_{1}\right), \ldots,\left(v<e_{n}\right)\right)$ is a circuit if $e_{0}=e_{n}$. Let $\mathscr{A}_{v}$ denote the set of lines of $\mathscr{A}$ which contain $v$. A circuit $\gamma=((v<$ $\left.\left.e_{0}\right),\left(v<e_{1}\right), \ldots,\left(v<e_{n}\right)\right)$ is full if, for every $l \in \mathscr{A}_{v}$, there exist at least two distinct indices $1 \leq j<k \leq n$ such that $\left|e_{j}\right|=\left|e_{k}\right|=l$. A system of weights $\Omega: \operatorname{Corn}(\mathscr{A}) \rightarrow \mathbf{R}^{+}$is said to be $\mathscr{A}$-admissible if, for every $v \in \Gamma^{(0)}(\mathscr{A})$ and every full circuit $\gamma$ of $\Lambda_{v}$, we have $\Omega(\gamma) \geq 2 \pi$.

Let $f \in \Gamma^{(2)}(\mathscr{A})$. We denote by $d(f)$ the number of vertices $v \in \Gamma^{(0)}(\mathscr{A})$ such that $v<f$. It is also the number of edges $e \in \Gamma^{(1)}(\mathscr{A})$ such that $e<f$. For a given system of weights $\Omega: \operatorname{Corn}(\mathscr{A}) \rightarrow \mathbf{R}^{+}$, we define the weight of $f$ to be

$$
\Omega(f)=\sum_{v<f} \Omega(v<f) .
$$

A system of weights $\Omega$ : $\operatorname{Corn}(\mathscr{A}) \rightarrow \mathbf{R}^{+}$is said to be aspherical if, for every $f \in \Gamma^{(2)}(\mathscr{A})$, we have $\Omega(f) \leq(d(f)-2) \pi$.

Theorem 3 (Falk [Fa]). If $\Gamma(\mathscr{A})$ admits a system of weights which is $\mathscr{A}$ admissible and aspherical, then $\mathscr{A}$ is a $K(\pi, 1)$ arrangement.

Remark. There is a similar criterium given in [JS] for a real arrangement of lines to be $K(\pi, 1)$.

2.2. Proof of Theorem 2. Let $\Pi=\left(\Pi_{1}, \Pi_{2}\right)$ be a factorization of $\mathscr{A}$. Let $(v<f)$ be a corner of $\Gamma(\mathscr{A})$. Let $e_{1}$ and $e_{2}$ be the two edges of $\Gamma(\mathscr{A})$ such that $v<e_{i}<f(i=1,2)$. We say that $(v<f)$ is coloured if, up to some permutation, $\left|e_{1}\right| \in \Pi_{1}$ and $\left|e_{2}\right| \in \Pi_{2}$. We consider the system of weights defined by

$$
\Omega(v<f)= \begin{cases}\frac{\pi}{2} & \text { if }(v<f) \text { is coloured }, \\ 0 & \text { otherwise. }\end{cases}
$$

Let $v \in \Gamma^{(0)}(\mathscr{A})$. Let $\gamma=\left(\left(v<e_{0}\right),\left(v<e_{1}\right), \ldots,\left(v<e_{n}\right)\right)$ be a full circuit of $\Lambda_{v}$. For $j=1, \ldots, n$, let $\left(v<f_{j}\right)$ be the edge of $\Lambda_{v}$ incident with $\left(v<e_{j-1}\right)$ and $\left(v<e_{j}\right)$. By definition of a factorization, we may assume that $\mathscr{A}_{v} \cap \Pi_{1}$ is a singleton $\left\{l_{0}\right\}$. By definition of a full circuit, there exist two indices $1 \leq j<k \leq n$ such that $\left|e_{j}\right|=\left|e_{k}\right|=l_{0}$. Obviously, $j \neq k-1$ and $k \neq j-1$ (we assume that $j-1=n$ if $j=1)$, and $\left(v<f_{j-1}\right),\left(v<f_{j}\right),\left(v<f_{k-1}\right)$, and $\left(v<f_{k}\right)$ are coloured corners. Thus,

$$
\Omega(\gamma) \geq \Omega\left(v<f_{j-1}\right)+\Omega\left(v<f_{j}\right)+\Omega\left(v<f_{k-1}\right)+\Omega\left(v<f_{k}\right)=2 \pi .
$$

This shows that $\Omega$ : $\operatorname{Corn}(\mathscr{A}) \rightarrow \mathbf{R}^{+}$is $\mathscr{A}$-admissible.

Let $f \in \Gamma^{(2)}(\mathscr{A})$. If $f$ is a triangle (i.e., $d(f)=3$ ), then there exist at most two vertices $v_{1}, v_{2} \in \Gamma^{(0)}(\mathscr{A})$ such that $v_{i}<f$ and the corner $\left(v_{i}<f\right)$ is 
coloured (for $i=1,2$ ). Thus,

$$
\Omega(f) \leq 2 \cdot \frac{\pi}{2}=(d(f)-2) \pi .
$$

If $d(f) \geq 4$, then

$$
\Omega(f) \leq d(f) \frac{\pi}{2} \leq(d(f)-2) \pi .
$$

This shows that $\Omega: \operatorname{Corn}(\mathscr{A}) \rightarrow \mathbf{R}^{+}$is aspherical.

\section{REFERENCES}

[De] P. Deligne, Les immeubles des groupes de tresses généralisés, Invent. Math. 17 (1972), 273302.

[Fa] M. Falk, $K(\pi, 1)$ arrangements, Topology (to appear).

[FJ] M. Falk and M. Jambu, Factorizations and colorings of combinatorial geometries, preprint, 1989.

[FR] M. Falk and R. Randell, On the homotopy theory of arrangements, Complex Analytic Singularities, Adv. Stud. Pure Math., vol. 8, North-Holland, Amsterdam, 1987, pp. 101-124.

[Ja] M. Jambu, Fiber-type arrangements and factorization properties, Adv. Math. 80 (1990), $1-21$.

[JS] T. Januszkiewicz and J. Swiatkowski, On the asphericity of plane arrangements, preprint, 1991.

[OS] P. Orlik and L. Solomon, Combinatorics and topology of complements of hyperplanes, Invent. Math. 56 (1980), 167-189.

[OT] P. Orlik and H. Terao, Arrangements of hyperplanes, Springer-Verlag, New York, 1992.

[Te1] H. Terao, Modular elements of lattices and topological fibration, Adv. Math. 62 (1986), 135-154.

[Te2] _ Factorizations of Orlik-Solomon algebras, Adv: Math. 91 (1992), 45-53.

Département de Mathématiques, Université de Nantes, 2 rue de la Houssiniere, 44072 Nantes Cedex 03, France

Current address: Département de Mathématiques, Université de Bourgogne, B.B. 138, 21004 Dijon Cedex, France

E-mail address: lparisesatie.u-bourgogne.fr 